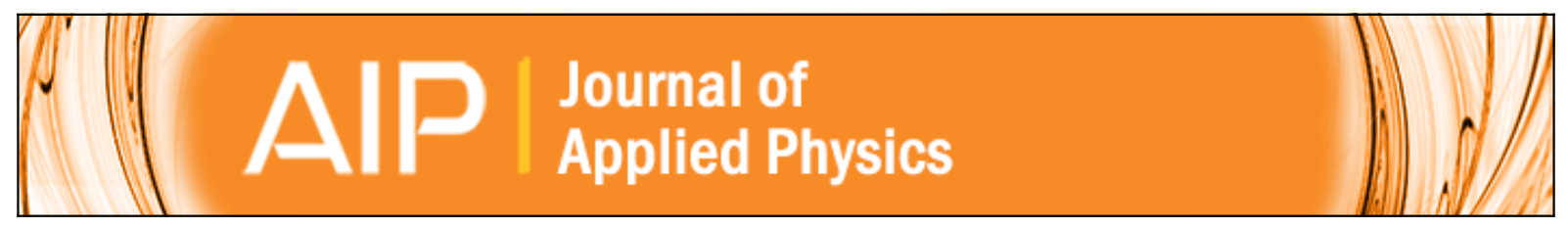

\title{
New results in the theory of wetting transitions (invited)
}

D. M. Kroll

Citation: Journal of Applied Physics 61, 3595 (1987); doi: 10.1063/1.338686

View online: http://dx.doi.org/10.1063/1.338686

View Table of Contents: http://scitation.aip.org/content/aip/journal/jap/61/8?ver=pdfcov

Published by the AIP Publishing

\section{Articles you may be interested in}

An introduction to inhomogeneous liquids, density functional theory, and the wetting transition

Am. J. Phys. 82, 1119 (2014); 10.1119/1.4890823

Theory of the forced wetting transition

Phys. Fluids 24, 072104 (2012); 10.1063/1.4736531

New Results on Nucleon Resonance Transition Form Factors

AIP Conf. Proc. 1182, 900 (2009); 10.1063/1.3293954

New results for phase transitions from catastrophe theory

J. Chem. Phys. 120, 11090 (2004); 10.1063/1.1740756

Wetting transitions in polymer blends: Comparison between simulation and theory

J. Chem. Phys. 105, 3849 (1996); 10.1063/1.472205

\section{MIT LINCOLN}

\section{LABORATORY} CAREERS

Discover the satisfaction of innovation and service

to the nation
- Space Control

- Air \& Missile Defense

- Communications Systems \& Cyber Security

- Intelligence, Surveillance and Reconnaissance Systems
- Advanced

Electronics

- Tactical Systems

- Homeland

Protection

- Air Traffic Control

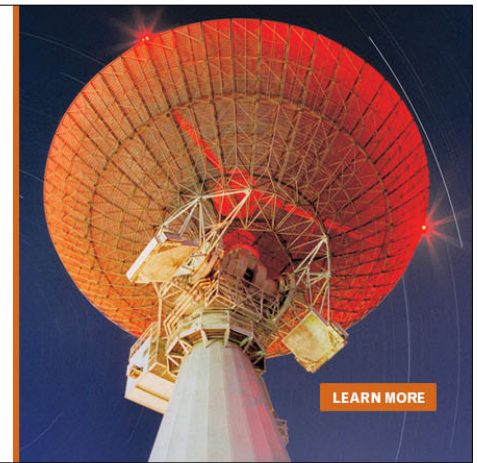




\section{New results in the theory of wetting transitions (invited)}

D. M. Kroil

Physics Department, Virginia Polytechnic Institute and State University, Blacksburg, Virginia 24061 and Institut für Festkörperforschung, Rernforschungsanlage Jülich, 5170 Jüich, West Germany ${ }^{\text {à }}$

The aim of this contribution is to draw attention to some recent developments in the theory of wetting transitions which may be of interest to the magnetism community. After presenting a qualitative discussion of wetting phenomena, the predictions of both the mean field and renormaiization group ( $R G$ ) theories of wetting with short range forces are reviewed. Recent Monte Carlo (MC) results are also described and possible causes of some of the discrepancies between the $\mathrm{RG}$ and $\mathrm{MC}$ results are discussed. Wetting phenomena in magnetic systems with a first-order bulk transition are considered and it is shown that metallic magnets and magnetic insulators (with short-range exchange) belong to different universality classes. Both the need for and the feasibility of experimental tests of the theory are emphasized.

\section{MTRODUCTION}

The theoretical description of the wetting transition proposed by $\mathrm{Cahn}^{2}$ and developed by numerous authors ${ }^{3}$ is based on the simple phenomenological free energy functional

$$
\begin{aligned}
F= & \int d V\left[\frac{1}{2}(\nabla \phi)^{2}-(\tau / 2) \phi^{2}+(g / 4 !) \phi^{4}-h \phi\right] \\
& +\int d S\left[(c / 2) \phi^{2}+h_{1} \phi\right]
\end{aligned}
$$

for a scalar field $\phi$, where the integrals extend over the volume $V$ and the surface $S$, respectively. For the semi-infinite geometry normally considered, $S$ is the plane $z=0$ and $V$ is the half-space $z \geqslant 0$.

The bulk terms in (1) are the familiar ones that appear in the usual Landau expansion of the free energy of a single component system. In particular, $\tau$ (the temperature) is a measure of the distance from the bulk critical point $r>0$ corresponds to the ordered phase and $h=0, \tau>0$, defines the coexistence piane.

The effect of the wall is described by the surface temperature $c$ and the surface magnetic field $h_{1}$. In particular, if the bulk boundary conditions far from the wall favor one phase (for example, $\phi>0$ ), and if $h_{1}$ is positive, then at sufficiently low temperatures a layer of down spins with a finite thickness (determined by $h_{1}, c_{9}$ and $\tau$ ) may form at the surface. Werting occurs if the thickness of this adsorbed layer diverges at some higher temperature $T_{w}$.

This model is easily analyzed in the mean-field (MF) approximation. The extremal solution of $(1)$ is determined from

$$
\delta F /\left.\delta \phi(r)\right|_{m}=0,
$$

where $m=\langle\phi\rangle$. The surface term in (2) leads to the boundary condition

$$
\dot{m}(0)=c m(0)+h_{1} .
$$

Extremal solutions depend only on the coordinate $z$ perpendicular to the suriace.

") Permanent address.
Consider now $h=0, \quad h_{1}>0, \quad$ and assume $m(z) \rightarrow m_{b}=\sqrt{6 \tau / g}$ for $z \rightarrow \infty$. A first integral of (2) then yiclds

$$
\dot{m}^{2}(z)=2\left\{U\left(m_{b}\right)-U[m(z)]\right\}
$$

with

$$
U(m)=(\tau / 2) m^{2}-(g / 4 !) m^{4}+h m .
$$

Representing Eq. (4) graphically, plotting $\dot{m}$ vs $m$ as in Fig. 1 , we obtain two parabolas. The boundary condition (3) is represented by the straight line $A$. For the chosen boundary conditions $m(z)$ is increasing from the wall to infinity. The solution starts at $z=0$ at the intersection of $A$ with the solid curve. For $\tau$ large enough ( $T$ small enough) $A$ will intersect the rrajectory as indicated at some $m>-m_{b}$. If the slope of $A$ is larger than the slope of line $B$, the tangent at the point $\left(m=-m_{b}, \dot{m}=0\right)$, there is a single intersection of $A$ with the relevant parabola. This occurs for $c>\sqrt{2} \overline{2}$, which is the condition for having a critical wetting transition. If the temperature is raised in this case, $\tau$ and $m_{b}$ decrease; assuming $h_{1}$ and $c$ remain fixed, the fixed point at $-m_{b}$ will move towards the line $A$. When it reackes $A$ the thickness of the wetting layer diverges. This occurs when $-c n_{b}+h_{1}$ van-

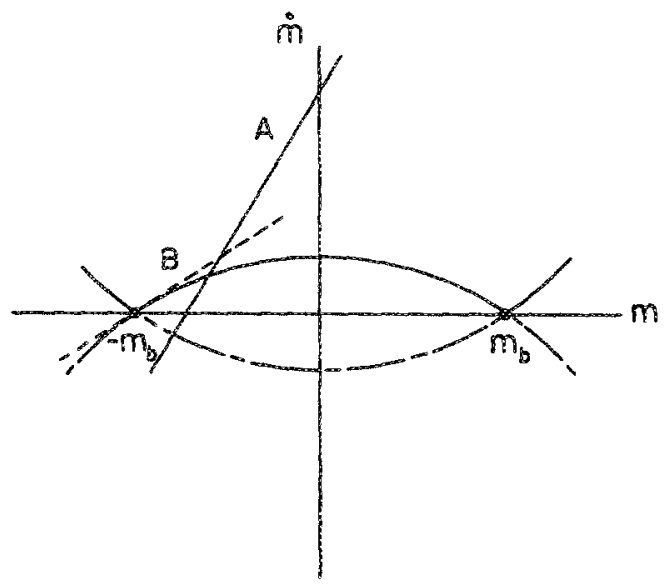

FIG $1 . m$ as a function of $m$. The wall boundary condition is the straight line $A$. The dashed line $B$ rangent at $i n=0, m=-m_{b}$ is the separatrix between second-order and first-order transitions. 
ishes, i.e., for $\tau=\tau_{\omega}=g h_{1}^{2} /\left(6 c^{2}\right)$. For temperatures between $T_{w}$ and $T_{c}$ (the bulk critical temperature) the thickness of the down-spin layer remains infinite and a finite positive bulk magnetic field $h$ is nocessary to keep the interface within any specified finite distance of the wall.

For $h=0$ and $T<T_{w}$ the explicit solution of (2) is

$m(z)=m_{b} \tanh \left[\sqrt{\tau / 2}\left(z-z_{0}\right)\right]$,

with $z_{0}$ determined by substituting (5) in (3). The surface free energy density is given by

$$
\begin{aligned}
\Sigma= & \int_{0}^{\infty} d z\left\{\frac{1}{2} \dot{m}(z)^{2}-(\tau / 2)\left[m(z)^{2}-m_{b}^{2}\right]\right. \\
& \left.+(g / 4 !)\left[m(z)^{4}-m_{b}^{2}\right]\right\}+\frac{1}{2} c m_{s}^{2}+h_{1} m_{s} \\
= & \sqrt{g / 12}\left(m_{s}^{3}-3 m_{b}^{2} m_{s}+2 m_{b}^{3}\right) / 3+\frac{1}{2} c m_{s}^{2}+h_{1} m_{s},
\end{aligned}
$$

where $m_{s}=m(0)$ is the magnetization at the wall. The MF condition for determining $z_{0}$ is nothing but $\partial \mathbf{\Sigma} / \partial m_{s}=0$. The influence of finite bulk field $h$ can be studied perturbatively. A scaling form for the singular part of the surface free energy density $\Sigma_{s}$ is easily derived. In particular, one finds

$$
\Sigma_{s} \sim t^{2} \Omega_{1}\left(h / t^{2}\right)+h \operatorname{in}\left[t^{2} \Omega_{2}\left(h / t^{2}\right)\right]
$$

at the critical wetting transition, where $t$ is the reduced temperature (both $\tau$ and $h_{1}$ have projections on $t$ ). Quite generally $\Sigma_{s}$ has the scaling form ${ }^{4}$

$$
\Sigma_{s} \sim t^{2-\alpha} \Omega\left(h / t^{\Delta}\right)
$$

so that $\alpha=0$ and $\Delta=2$ in the $M F$ approximation for this model. Further results of the MF approximation are:

(i) the correlations in directions parallel to the wall diverge with a characteristic length

$$
\xi_{p} \sim t^{-\cdots v_{p}}=t^{-1}
$$

so that $v_{p}=1$, and

(ii) the coverage, i.e., the distance of the interface from the wall, diverges as

$$
z_{0} \sim \partial \Sigma_{s} /\left.\partial h\right|_{h=0} \sim-\ln (t) \text {. }
$$

The hyperscaling relation $2-\alpha=D v_{p}$ is satisfied for $D_{c}$ (interface dimension) $=2$, i.e., bulk dimension 3. It is therefore not surprising that the upper critical dimension above which MF theory is valid is $d_{c}=3$.

The fluctuations which are responsible for the vioiation of the MF approximation in $d \leqslant 3$ are capillary waves. ${ }^{5,6}$ It therefore makes sense to construct an effective interface model in which the dynamical variable is the position $l(\bar{\rho})=z_{0}+\zeta(\bar{\rho})$ of the interface. Projecting out the relevant fluctuations, we have

$$
\phi(z, \bar{\rho})=m(z)-\dot{m}(z) \xi(\bar{\rho}) \approx m[z-\xi(\bar{\rho})] .
$$

Inserting (9) in (1) we obtain a free energy functional of the form

$$
F\{l\}=\int d^{d-1} \rho\left[(\sigma / 2)(\nabla l)^{2}+\Sigma(l)\right]
$$

where $\Sigma(h)$ is obtained by substituting $(9)$ in $(6)$. For the isotropic system studied here, $\sigma$ is the surface tension of the free interface. For uniform $l, \Sigma(l)$ reduces to the free energy per unit area (6). Indeed, as already mentioned, the location of the interface in the MF approximation is determined by minimizing $\Sigma(l)$ with respect to $l$. Near $T_{w}$ the interface is far from the wall and an expansion of $\Sigma(l)$ for large $l$ yields ${ }^{\circ}$

$$
\Sigma(i)=-A e^{-l / \xi_{b}}+B e^{-2 l / \xi_{b}}+C e^{-3 l / \xi_{b}}+\ldots
$$

for $h=0$, where

$$
\begin{aligned}
& A=2 m_{b}\left(c m_{b}-h_{1}\right), \\
& B=2 m_{b}^{2}(c-\sqrt{2 r})+A, \\
& C=2 m_{b}\left[(4 / 3) m_{b} / \xi_{b}-3 c m_{b}+h_{1}\right],
\end{aligned}
$$

and $\xi_{b}=1 / \sqrt{2} \tau$ is the bulk correlation length. The MF phase diagram is easily reproduced using this result. The critical wetting line corresponds to $A=0, B>0$, and $T<T_{w}$ corresponds to $A>0$. The first-order line corresponds to $B<0, B=-4 A C$.

Renormalization group ( $R G$ ) treatments of the critical wetting transition for model (1) start with the effective interface model $(10), 5,7,8$ The essential ingredients of the $R G$ treatments are: (i) a convolution of $\Sigma(l)$ with the capillary wave excitations, and (ii) a normal ordering of the resulting effective Hamiltonian. A very clear way of accomplishing this program is the functional $R G$ procedure ernployed by Fisher and Huse." The resulting critical behavior is predicted to differ dramatically from the MF result. For example, while the MF approximation predicts that for $h=0$ the correlation length $\xi_{p}$ describing correlations in the interface diverges with the exponent $v_{p}=1$, recent $R G$ work predicts that $v_{p}$ is nonuniversal in $d=3$, depending on the value of $\omega=k_{B} T /\left(4 \pi \sigma \xi_{b}^{2}\right)$. For $\omega<2$, the wetting transition is predicted to occur when the strength $A$ of the attractive tail of the interface-wall potential goes to zero. In both regime 1 , $0<\omega<\frac{1}{2}$, and regime $\mathrm{II}, \frac{1}{2}<\omega<2$, the film thickness $\langle l\rangle$ diverges logarithmically as the wetting transition is approached $\left(A \rightarrow A_{c}=0\right)$ and the correlation length $\xi_{p}$ diverges as a power of the deviation from wetting $t \sim\left(A-A_{c}\right)$ (with logarithmic corrections in regime II). The critical exponents vary with $\omega$ and are nonanalytic at the multicritical point at $\omega=\frac{1}{2}$. For $\omega>2$, regime $\mathrm{MI}$, the wetting transition occurs at finite strength, $A_{c} \neq 0$, of the wall attraction. The film thickness diverges as $1 / t$ and the correlation length di-

\begin{tabular}{|c|c|c|}
\hline $0<\omega<\frac{1}{2}$ & $\xi_{p}=\xi_{0} t^{-v_{r}}$ & $v_{p}=(1-\omega)^{-\frac{1}{1}}$ \\
\hline$\frac{1}{2}<\omega<2$ & $\xi_{P}=\xi_{0^{i}}{ }^{-v_{P}}|\ln t|^{-i}$ & $\begin{array}{c}v_{P}=\left(V / 2-V(\omega)^{-2}\right. \\
\tilde{\nu}=\sqrt{\omega / 8} v_{P}\end{array}$ \\
\hline $2<\omega$ & $\xi_{P} \sim e^{-x^{t-1} \mid \operatorname{in} x i}$ & $c=$ const \\
\hline
\end{tabular}
verges as $e^{1 / t}$ in this regime. Near the multicritical point at $\omega=2, A_{c}$ vanishes as $\omega-2$ and for a large range of $t$, the behavior will be dominated by the multicritical point, at which $\langle l\rangle \sim 1 / t^{2}$ and $\xi_{p} \sim e^{-1 / t^{2}}$. ${ }^{8}$ The predicted behavior is summarized in Table $\bar{I}$.

Before proceeding to a discussion of more recent developments, we recall another manifestation of wetting which is relevant to the subsequent discussion. Making the substitu-

TABLE I. Renormalization group predictions for the critical behavior of the interface correlation length $\xi_{p}$ for model (10) in three dimensions. The correlation length exponent $v_{p}$ depends explicitly on the ratio $\omega=k_{B} T /$ $\left.\left(4 \pi \sigma \xi_{h}^{2}\right)\right|_{T_{w}}$. 
tion $\phi(r) \rightarrow \eta(r)+m_{b}$ in (2) we arrive at a free energy functional for $\eta(r)$ of the form

$$
\begin{aligned}
F\{\eta\}= & \int d V\left[\frac{1}{2}(\nabla \eta)^{2}+r \eta^{2}-v \eta^{3}+u \eta^{4}\right] \\
& +\int d S\left[(c / 2) \eta^{2}+H_{1} \eta\right]
\end{aligned}
$$

where $H_{1}=-c m_{b}+h_{1}$. The bulk free energy density in (11) has a cubic invariant. It describes a system with a firstorder transition. The critical wetting transition discussed above occurs for $h=0$ at $-c m_{b}+h_{1}=0$. However, $h=0$ is the position of the first-order transition in (11) and $-c m_{b}+h_{1}=0$ implies $H_{1}=0$. In this form the critical wetting transition is sometimes referred to as a surface-induced disorder transition. ${ }^{9,10}$ It can occur in semi-infinite systems with a free surface: as the bulk transition temperature is approached from below, a narrow region of the hightemperature disordered phase intervenes at the free surface and finally wets it at the bulk transition temperature, where the ordered and disordered phases coexist. At this transition the order at the surface goes continuously to zero. Such behavior appears to be quite common and will be discussed in more detail below. It has been observed at the order-disorder transition in $\mathrm{Cu}_{3} \mathrm{Au}$ (Ref. 11) and a closely related phenomena is the surface melting of certain crystals as the solidliquid-phase boundary is approached. ${ }^{12}$ We expect such behavior to occur in many semi-infinite magnetic systems with a first-order transition.

One very attractive aspect of this wetting transition is the possibilities it offers for the experimentalist. In the classicai wetting experiments in binary mixtures it is generally only possible to measure "excess" quantities such as the coverage. Furthermore, the relaxation times involved in many of these experiments are extremely long, making it difficult to make true equilibrium measurements. On the other hand, at surface induced disorder transitions it is possible to probe the behavior of local quantities such as the surface order parameter using LEED, SPLEED, or by performing total reffection experiments using synchrotron radiation or neutrons. In this context, total reffection experiments are particularly attractive since they provide information concerning both local and excess quantities. In addition, their interpretation is unambiguous. ${ }^{13}$

The previous discussion reviews several of the older theoretical results. One of the most intriguing results were the $R G$ predictions for the critical wetting exponents. Recently Monte Carlo (MC) methods have been used to study wetting in a simple cubic Ising model with nearest-neighbor interactions in order to test these predictions. ${ }^{14}$ The critical behavior was found to be consistent with MF theory; the singularities predicted by $R G$ treatments were not observed! Subsequently, the effective interface model $(10)$ was simulated and results were obtained which agree with the RG work. ${ }^{15}$ This work is reviewed and discussed in Sec. II. The results discussed in Sec. II make it clear that our understanding of wetting in systems with short-range forces in $d=3$ is still incomplete. It would thus be of considerable interest to study such behavior experimentally. This may be possible in magnetic insulators with a bulk first-order phase transition.
For this reason, the order-disorder transition at the surface of a face-centered-cubic ( $\mathrm{fcc}$ ) Ising antiferromagnet is discussed in Sec. III. Finally, in Sec. IV we review what is known about the universality classes for critical wetting, discuss briefty wetting in ordering alloys with long-range interactions and metallic magnets.

\section{I1. MONTE CARLO STUDY OF CRITICAL WETTNG WITH SHORT-RANGE EORCES}

A nearest-neighbor Ising model on a simple cubic lattice was studied by Binder, Landau, and Kroll. ${ }^{14} \mathrm{~A}$ thin film $L \times L \times D$ geometry with two free (100) surfaces was employed. A film thickness $D=40$ layers was choser; this was sufficient in order to avoid inite size effects associated with finite $D$. Periodic boundary conditions in the directions parallel to the surface were used and $L$ was varied between $L=10$ and $L=50$ in order to study the finite size behavior as a function of $L / \xi_{F}$. Sampling techniques with preferential surface site selection were employed.

Denote the exchange constants by $J$ in the bulk and $J_{s}$ on the surface, and the surface field by $h_{1}$. Depending on the parameters $J / k_{B} T$ and $J_{s} / J_{s}$ the wetting transition was found ${ }^{16}$ to be either first order or continuous, in agreement with theory. ${ }^{4}$ The simulations were performed at temperatures $T$ such that $J / k_{B} T \geqslant 0.25\left(J / k_{\mathrm{B}} T_{C}=0.22169\right)$ in order to avoid crossover to bulk critical phenomena, but $J / k_{B j} T \leqslant 0.35$ in order to avoid "layering" (for $T<T_{R}$, the roughening temperature, "multilayer adsorption" occurs).

In order to compare the results for the critical exponents with theory, the value of $\omega\left(=k_{B} T / 4 \pi \sigma \xi_{b}^{2}\right)$ for the Ising model in the relevant remperature regime is needed. Near $T_{c}$, $\omega$ tends to a universal value $\omega_{c} \approx 1.2+0.3 .{ }^{17}$ For $T<T_{c}$, $\sigma$ shoula be identified with the "surface stiffness". ${ }^{18}$ Unfortunately, $\sigma$ is not known for the Ising model; only $f_{\text {int }}$, the interfacial free energy density of a planar (100) interface, has been estimated. ${ }^{19}$ It is known, however, that $\sigma$ exhibits a universal jump from $\sigma a^{2} / k_{B} T_{R}=\pi / 2$ (where $a$ is the spacing of the bulk crystalline planes normal to the interface) for $T \rightarrow T_{R}^{+}$to $\sigma=\infty$ for $T=T_{R}^{-}$. Using the estimate of Tarko and Fisher ${ }^{20}$ for $\xi_{b}$ this leads to a value of $\omega_{R}$ between 0.8 and 1.25 for the range of values of $T_{R}$ suggested in $R$ Ref. 19 (b). This is the same order as $\omega_{c}$ so that $\omega$ is probably of order one at the temperatures employed in the simulation. Note that below $T_{R}$ capillary wave excitations are suppressed so that $\omega=0$.

The results are the following ${ }^{14}$ : The surface excess (or coverage) was found to diverge logarithmically in $t \sim\left(h_{1}-h_{1 c}\right)$ at the critical wetting transition as well as logarithmically in $h$ for $T>T_{w}$ as coexistence is approached (complete wetting). These results are in agreement with theory. However, this logarithmic behavior is predicted by both the $R G$ work and MF theory. Since a direct evaluation of the correlation length is technically difficult, the singular part of the surface magnetization at the transition $\Delta m_{1}=m_{1}-m_{1}(h=0)$ and $\chi_{1}=\partial m_{1} / \partial h$ were calculated. From scaling we expect ${ }^{21}$

$$
\Sigma_{s}=t^{2 v_{r}} \Omega\left(h / t^{2 v_{p}}\right)
$$


at the critical wetting transition, where $t=h_{1}-h_{1 c}$. This implies

$$
\chi_{1}=\partial^{2} \Sigma_{s} / \partial h \partial h_{1}=t^{-1} \chi_{1}\left(h t^{\cdots 2 v_{p}}\right)_{t=0}^{\rightarrow h^{-1 / 2 v_{p}}}
$$

Similarly, we have $\Delta m_{1} \sim h^{1-1 / 2 v_{p}}$. Surprisingly, for both $J /$ $k_{B} T=0.25$ and 0.35 the results are consistent with $v_{p}=1$ (Fig. 2), i.e., the MF result instead of the (nonuniversal) exponent listed in Table I. As discussed above, since we believe $\omega>\frac{1}{2}$ at these temperatures (so that $v_{p}>2$ ), these results are in striking disagreement with the theoretical predictions?

Subsequentiy, Gompper and Kroll15 simulated lattice version of the effective interface model

$$
\begin{aligned}
F= & \int d^{2} \rho\left[(\sigma / 2)(\nabla l)^{2}-A e^{-l / \xi_{b}}\right. \\
& \left.+B e^{-2 l / \xi_{b}}+h l\right], \quad l>0,
\end{aligned}
$$

directly. Since both the MF approximation as well as the $R G$ theories predict that $\xi_{p}$ diverges as $h^{-1 / 2}$ for $h \rightarrow 0$ at the critical wetting transition, it was convenient to simulate the singular $h$ dependence of the coverage and excess surface magnetization $\Delta m_{1}\left[\right.$ given by $\left.\left\langle e^{-l / \xi_{b}}\right\rangle\right]$. AnL $\times L$ geometry with periodic boundary conditions was chosen and $L$ was varied between 10 and 40 in order to study finite size effects.

Simulations were performed for two values of $\omega(0.25$ and 1.0 ). For $\omega=1.0, B=1.0$ was employed, while for $\omega=0.25$ simulations were performed for both $B=0.1$ and $1.0 \mathrm{in}$ order to determine the influence of $B$ on the size of the asymptotic critical region. For $\omega<\frac{1}{2}$ theory $y^{8}$ predicts that the coverage $\langle l\rangle$ diverges asymptotically as $(1+2 \omega) \ln \xi_{p}$ with $\xi_{p} \sim h^{-1 / 2}$ while for $\frac{1}{2}<\omega<2$, $\langle l\rangle$ $\sim \sqrt{8} \omega\left[\ln \xi_{p}-(1 / 8) \ln \ln \xi_{p}\right]$. In both cases we expect $\Delta m_{1} \sim\left\langle e^{-1 / 5_{b}}\right\rangle \sim h^{1-1 / 2 v_{p}}$. The results of the simulation are shown in Fig. 3. The solid lines are obtained by integrating the full functional $R G$ recursion relations and matching in the usual fashion when the long wavelength fuctuations

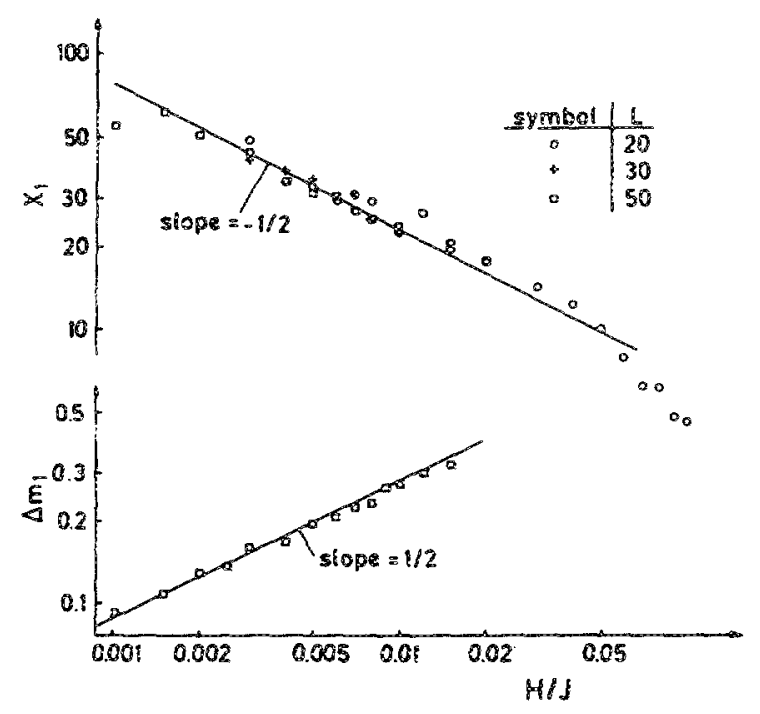

FIG. 2. Surface-layer susceptibility $\chi_{3}$ for $J_{s} / J=1, J / \lambda_{B} T=0.35$, and $h_{1} / J=-0.89$, and excess surface magnetization $\Delta m_{1}$ for $J_{5} / J=1$, $J / k_{B} T=0.25$, and $h_{1} / J=-0.55$, plotted $v s h / d$.

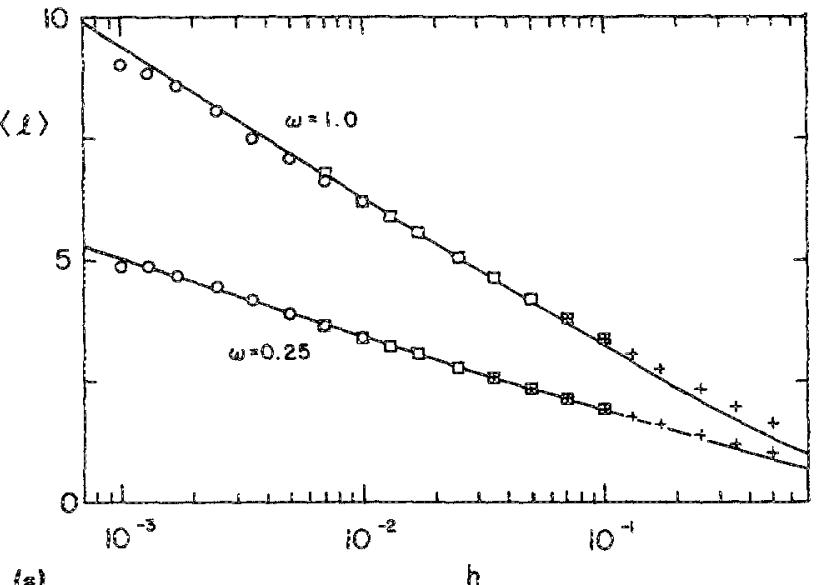

(a)

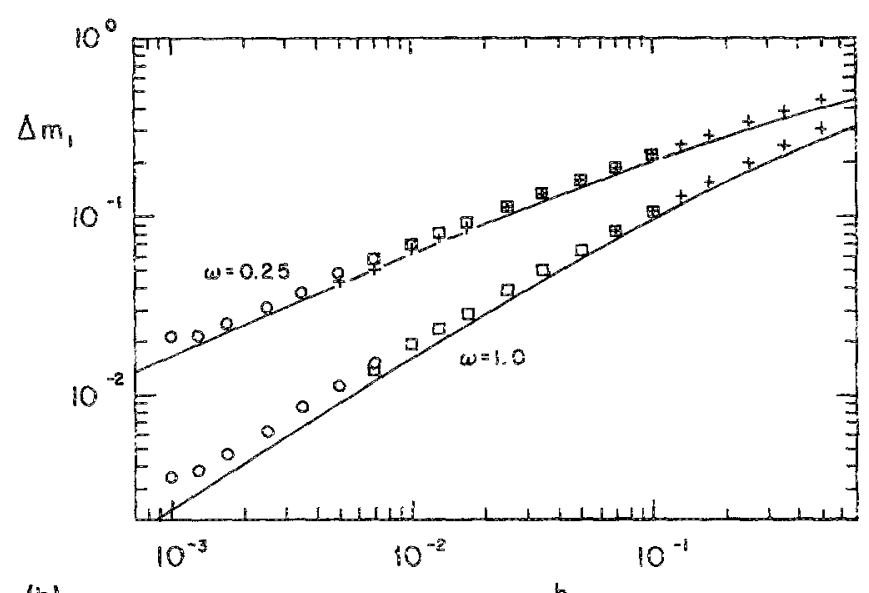

(b)

h

FIG. 3. (a) Coverage $\langle l\rangle$ and (b) excess surface magnetizaiton $\Delta m_{1}$ vs $h$ for (1) $\omega=0.25, B=0.1$ and (2) $\omega=1, B=1$. The solid lines are obtained by integrating the full $R G$ recursion relations.

have been integrated out. The agreement is excellent! This shows that the RG treatments of (10) are correct, at least in the range of $\omega$ investigated.

The discrepancy between these results and the simulation of the full three dimensional Ising model is puzzling. Several explanations are possible. It may be that the value of $\omega$ for the Ising model is much smaller than we believe at the temperatures tested. It's also possible that the critical region is extremely narrow and has not yet been reached in the three-dimensional simulation. This, however, seems unlikely since the range of scaling fields studied in the two simulathons is about the same. It is also possible that there is something fundamental which we do not yet understand and that the effective interface model (10) does not captivate the essential physics of wetting in the three dimensional Ising model.

In any case, these results indicate that the present theoretical understanding of critical wetting with short-range forces in $d=3$ is still incomplete. More work is needed to determine the width of the asymptotic critical regime, to understand the influence of lattice effects, and to narrow down uncertainties in the value of $\omega$ for the Ising model. 


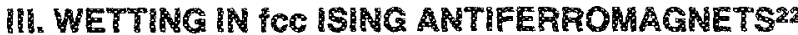

Help from the experimentalist would be most useful in sorting out these problerns. As already mentioned, magnetic insulators with a bulk first-order transition are good candidates for such experiments. Metamagnets, ${ }^{23}$ i.e., Ising antiferromagnets which undergo a first-order transition in suffciently strong external magnetic fields are a common example of such systems. Another example, which we discuss here in more detail, is an ling antiferromagnet on an fcc lattice. In this case the Landau-Ginzburg model describing the transition to the phase with $L 1_{2}$ structure contains a cubic invarient so that the transition is intrinsically first order.

A common characteristic of all these systems is that several densities are needed to describe the order. The prevailing wisdom has been that the resulting wetting behavior is independent of the number of densities needed to describe the thermodynamic state. This belief amounts to the assumption that the only relevant length scale in the problem is that dictated by the order parameter. However, since this length remains finite at the transition (for finite range forces), this assumption is not justified in general. In fact, as we shall see, MF theory predicts that the wetting exponents are nonuniversal in this case, depending on many details of the system such as the structure of the ordered phase, the crystal surface, as well as the relative strength of the nearestneighbor ( $\mathrm{nn}$ ) and next-nearest-neighbor (nnn) interactions. This behavior is caused by the competition between at least two relevant length scales in the problem-that given by the order parameter and at least one of the nonordering densities. Before proceeding it should be emphasized that the novel features we discuss in this section are intimately connected with the short-range nature of the forces. The long-range forces induced by conduction electron in metallic alloys and magnets modify the critical behavior considerably; in particular, different critical exponents are to be expected and a nonuniversality of the type discussed in this section doesn't occur (see Sec. IV). On the other hand, the results should apply to magnetic insulators, in which exchange interactions are truly short range.

To illustrate this behavior, an fce Ising antiferromagnet with nn and nun interactions which exhibits an $l l_{2}$ ( $\left.\mathrm{Cu}_{3} \mathrm{Au}\right)$ groumd-state structure was studied. ${ }^{22}$ The Hamiltonian is of the form

$H=J \sum_{\langle\mathrm{nn}\rangle} \sigma_{i} \sigma_{j}-\alpha J \sum_{\langle\mathrm{nnn}\rangle} \sigma_{i} \sigma_{j}-H \sum_{i} \sigma_{i}-H_{i} \sum_{i=S} \sigma_{i}$,

where the first sum ranges over $\mathrm{n}$ and the second over $\mathrm{nm}$ pairs of sites $\alpha=0.2$ was used so that the nn interactions are antiferromagnetic and the nnn couplings ferromagnetic. The last term is a surface field acting on the first one or two surface planes. Surface enhancement effects were not considered.

Wetting transitions which occur at $(100),(110)$, and (111) free surfaces as the $L 1_{2}$ order-disorder transition is crossed going from the ordered to the disordered state were studied. In order to describe the ordered state we need to distinguish two types of sublattices. The $\alpha$ sublattice (preferred sites for "up" spins) was chosen to consist of all face centered lattice sites and the $\beta$ lattice to be composed of all corner sites of the fec lattice.

In the [111] direction all lattice planes are equivalent; two densities are thus needed to describe the wetting profiles. In contrast, in the [100] and [110] directions there are two distinct types of planes. Planes of type 1 consist of an equal number of $\alpha$ and $\beta$ sites, while the other, type 2 planes consist only of $\alpha$ sites.

In the MF approximation one finds that continuous wetting transitions are possible at all three surfaces for appropriately chosen surface fields $h_{i}$. In the [111] direction numerical solution of the lattice $M F$ equations indicates that the surface order goes to zero with the exponent $\beta_{1}=1$, in agreement with the single component model discussed in the introduction. Significantly different behavior was obtained at (100) and (110) surfaces. For a (100) surface one find $\beta_{1} \approx 2.22$ at the critical wetting transition while at a (110) surface, athough $\beta_{1}=\frac{1}{2}$, the true asymptotic scaling region is extremely narrow, indicating the presence of large correction to scaling terms.

The reason for the drastic difference in behavior at these three surfaces is that while one length scale (measured for example by the width of the MF order parameter interface) dominates in the [111] direction, there are two characteristic lengths of comparable size in the [100] and [110] directions. It's the competition between these two length scales which leads to this nonuniversal behavior. This effect is rather easy to understand in terns of an effective interface model similar to (10). A procedure similar to that outined above for the single component case leads to the free energy functional ${ }^{22}$

$$
\begin{aligned}
F= & \int d^{2} \rho\left[(\sigma / 2)(\nabla l)^{2}+A_{\eta} e^{-l / \xi_{\eta}}+A_{c} e^{-l / \zeta_{c}}\right. \\
& \left.+B_{\eta} e^{-2 l / \xi_{\eta}}+B_{c} e^{-2 l / \xi_{c}}+\ldots+t l\right]
\end{aligned}
$$

where $\xi_{\eta}\left(\xi_{c}\right)$ is the bulk order parameter (concentration) correlation length in the disordered surface phase in the direction perpendicular to the interface and the reduced temperature $t \sim\left(T^{*}-T\right)$ is a measure of the distance from the bulk first-order phase transition (located at $\left.T^{*}\right\}, A_{\eta}$ is proportional to the surface field conjugate to the order parameter [it's zero for model (12)] and $A_{c}$ is related to the surface fields $H_{i}$ in (12). The free energy functional (13) is derived in Ref. 22.

For $\xi_{\eta}>2 \xi_{c}$ the leading terms in (13) are $e^{l / \xi_{\eta}}$ and $e^{-2 l / \xi_{\eta}}$ so that the surface critical behavior is the same as in the single component case. However, qualitatively new behavior occurs for $\xi_{\eta}<2 \xi_{*}$. Consider $A_{\eta}=0$. Since the leading exponential term is $e^{-l / \xi_{c}}$, the transition for $t \rightarrow 0$ is continuous if $A_{c}>0$. The equilibrium thickness $\langle l\rangle$ of the wetting layer again grows logarithmically (but with a different amplitude), but the singular behavior of the surface order parameter is changed. In this case $e^{-\langle l\rangle / \xi_{c}} \sim t$ so that $\beta_{1}=\xi_{c} / \xi_{\eta}>\frac{1}{2}$. The exponent depends explicitly on the ratio of the length scales. ${ }^{24,25}$

In the [100] direction one finds $\xi_{c}>2 \xi_{\eta}$ so that $\beta_{1}>2$ in agreenent with the numerical results mentioned above. In the [110] direction $\xi_{\eta} \approx 2 \xi_{c}$ so that the asymptotic region is very narrow because of the competition between the two 
terms $e^{-l / \xi_{\mathrm{c}}}$ and $e^{--2 l / \xi_{\eta}}$ in (13).

Monte Carlo simulations were subsequently performed ${ }^{26}$ on model (12). (100) and (111) free surfaces were studied. A $2 L \times 2 L \times 2 D$ latice $(10 \leqslant L \leqslant 20$ and $10 \leqslant D \leqslant 23)$ was used in the simulation and preferential surface site selection was again employed. Between 7500 and $30000 \mathrm{MC}$ steps/spin were used in evaluating expectation vahes, and the reduced temperature interval $5 \times 10^{-3} \leqslant t \leqslant 10^{-1}$ was studied. In the [111] direction $\beta_{1}$ was found to depend rather strongly on the value of the surface field $H_{1}$ in this temperature range. This is consistent with the MF results. For large $H_{1}$ where $M F$ theory indicates that the critical region is widest, $\beta_{1} \rightarrow \frac{1}{2}$, the $M F$ exponent. In the [100] direction, $\beta_{1} \approx 1.78$ was found deep in the critical wetting region and $\beta_{1}^{*} \approx 0.76$ at the tricritical transition $\left[A_{c}=0 \mathrm{in}(12)\right]$. Since the value of $\omega$ is not known in this case it is not possible to compare these results directly with RG theory. However, MF theory predicts that $\beta_{1}^{2} / \beta_{1}=\frac{1}{2}$, while the $\mathrm{MC}$ data imply $\beta_{1} / \beta_{1} \approx 0.44$. Since $R G$ theory predicts that this ratio is an increasing function of $\omega$, the $\mathrm{MC}$ data again appear to support the MF prediction. As discussed in Sec. II, it is not yet clear why corrections to MF behavior were not observed.

\section{UNVERSALITY CLASSES AND LONG-RANGE FORCES}

Until now we have only considered short-range interactions. "Short range" in the current context means that the interactions drop off at least exponentially fast with distance. This condition is not fulfhed in many cases. For example, the dispersion forces which govern the wetting properties of fuids vanish as $r^{-6}$ at large distances. Similarly, the interactions in magnets with indirect or itinerate exchange are long range. In the rare earths, strongly localized $f$ electrons polarize the conduction electrons; this leads to RKKY interactions which are oscillatory and long range. The interactions in ordering alloys such as $\mathrm{Cu}_{3} \mathrm{Au}$ are of similar form.

The easiest way to understand the infuence of longrange interactions is to use an effective interface Hamilonian of the form (10). For dispersion forces the Hamiltonian has already been derived. ${ }^{27,28}$ Given the elementary interaction potentials, it would be easy to do so for ordering alloys or metallic magnets.

For long range interactions the potential $\Sigma(b)$ in $(10)$ is of the form

$$
\Sigma(l)=a_{0} l^{r_{0}}+a_{1} / l^{r}+\ldots+t l
$$

For dispersion forces $r_{0}=2$ and $r_{1}=3$. The critical wetting line corresponds to $a_{0}=0, t=0$, and $a_{1}>0$. Quite a lot is known concerning the critical behavior of the model defined by (10) and (14). We summarize here the results for dispersion forces; details may be found in Ref. 21 . In the MF approximation we have $\alpha=-1$ and $\Delta=4$. There are two upper critical dimensions, $d_{1}=11 / 5$ and $d_{0}=2$. For $d>d_{1}$, MF theory is correct, while for $d_{1}>d>d_{0}$ the transition still occurs at $a_{0}=0$, but the exponents are renormalized. In particular, $v_{p}=1 /[2(d-2)]$ and $v_{p} / \Delta=2 /(d+1)$. For $d<d_{0}$ the transition occurs at finite $a_{0}$; although the ratio $v_{p} / \Delta$ remains $2 /(d+1), v_{p}$ is not yet known for general $d$. These results have been verified by transfer matrix methods in $d=2 .^{21}$

The most important implication of these results is that since $d_{1}<3$ (for all long-range forces), MF theory should correctly describe the wetting behavior. Since the exponents $r_{0}$ and $\gamma_{1}$ in (14) depend sensitively on the basic interaction parameters, measurements of $\beta_{1}$ at surface induced disorder transitions could provide a useful check of our understanding of the magnetic or ordering interactions in these systems. This would be very important since the interaction parameters in real materiais are in general not well known.

\section{ACKNOWLEDGMENTS}

Enjoyable collaborations with K. Binder, G. Gompper, D. Landau, T. Meister, R. Lipowsky, and R. K. P. Zia are gratefully acknowledged.

'See, e.g., the review paper of D. E. Sullivan and M. M. Telo de Gama in Fluia Interfacial Phenomena, edited by C. A. Croxton (Wiley, New York, 1986).

25. W. Cahn, J. Chem. Phys. 66, 3667 (1977).

${ }^{3}$ See, e. g., R. Pandit and M. Wortis, Phys. Rev. B 25, 3226 (1982); $\mathbb{R}$. Pandit, Mi. Schick, and M. Wortis, Phys. Rev. B 26, 5112 (1982).

${ }^{4}$ H. Nakanishi and M. E. Fisher, Phys. Rev. Lett. 49, 1565 (1982),

${ }^{5}$ R. Lipowsky, D. M. Kroll, and R. K. P. Zia, Phys. Rev. B 27, 4499 (1983).

'D. Brézin, B. 1. Halperin, and S. Leibler, J. Phys. (Paris) 44, 775 (1983)

'D. Brézin, B. I. Fhalperin, and S. Leibier, Phys. Rev. Lett. 50, 1387 (1983).

${ }^{8}$ D. S. Fisher and D. A. Huse, Phys. Rev. B 32, 247 (1985).

${ }^{9}$ R. R. Lipowsky, Phys. Rev. Lett. 49, 1575 (1982); Z. Phys. B 51, 165 (1983).

${ }^{10} \mathrm{R}$. Lipowsky, J. Appl. Phys. 55, 2485 (1984).

"V. S. Sadaram, B. Farrel, R. S. Alben, and W. D. Robertson, Phys. Rev. Lett. 31, 1136 (1973); V. S. Sadaram, R. S. Alben, and W. D. Roberston, Sutf. Sci. 46, 653 (1974); E. G. McRae and R. A. Malic, Surf. Sci. 148, 551 (1984); K. D. Jamison, D. M. Lind, F. B. Dunning, and K. G. Walters, Surf. Sci. 159, L451 (1985); S. F. Alvarado, M. Campagna, A. Fattah, and W. Uelhof, Z, Phys. 866,103 (1987).

${ }^{12} \mathrm{~J}$. F. van der Veen and J. W. M. Frenken, Proceedings of ECOSS-8 (to appear in Surf. Sci. $)$.

S. Dietrich and H. Wagner, Phys. Rev, Lett. 51, 1469 (1983); Z. Phys. B $56,207(1984)$.

${ }^{14}$ K. Binder, D. P. Landau, and D. M. Kroll, Phys. Rev. Lett. 56, 2272 (1986).

${ }^{15} \mathrm{G}$. Gompper and D. M. Kroll (unpublished).

${ }^{16}$ K. Binder and D. P. Landau, J. Appl. Phys. 57, 3306 (1985); K. Binder, D. P. Landau, and D. M. Kroll, J. Magn. Magn. Mater. 54-57, 669 (1986).

${ }^{17}$ E. Brézin and S. Feng, Phys Rev. B 29, 472 (1984); K. K. Mon and D. Jasnow, Phys. Rev. A 30, 670 (1984); M. R. Moldover, Phys. Rev. A 3R, 1022 (1985).

${ }^{18}$ M. P. A. Fisher, D. S. Fisher, and J. D. Weeks, Phys. Rev. Lett. 48, 368 (1982).

19 (a) K. Binder, Phys. Rev. A 25, 1699 (1982); (b) E. Bürkner and D. Stauffer, Z. Pinys. B 53, 241 (1983).

${ }^{20}$ H. B. Tarko and M. E. Fisher, Phys. Rev. B 11, 1217 (1975).

${ }^{21}$ D. M. Kroll, R. Lipowsky, and R. K. P. Zia, Phys. Rev. B 32, 1862 (1985).

${ }^{22}$ D. M. Kroll and G. Gompper, Phys. Rev. B (to be published)

${ }^{23}$ E. Stryjiewski and N. Giordano, Adv. Phys. 26, 487 (1977).

${ }^{24} \mathrm{E}$. H. Hauge (Ref. 25 ) has obtained many of these results independently.

${ }^{25}$ E. H. Hauge, Prys. Rev. B 33, 3322 (1985).

${ }^{26} \mathrm{G}$. Gompper and D. M. Kroll (unpublished).

${ }^{27}$ S. Dietrich and M. Schick, Phys. Rev. B 31, 392 (1985).

${ }^{28}$ C. Ebner, W. F. Saam, and A. K. Sen, Phys. Rev. B 31, 6134 (1985). 